\title{
Over 400 million years of cooperation: Untangling the chondrocranium- dermatocranium connection
}

\author{
Susan M. Motch Perrine ${ }^{1, \&,{ }^{*}}$ \\ M. Kathleen Pitirri ${ }^{1, \&}$ \\ Emily L. Durham ${ }^{1}$ \\ Mizuho Kawasaki ${ }^{1}$ \\ Hao Zheng ${ }^{2}$ \\ Danny Z. Chen² \\ Kazuhiko Kawasaki ${ }^{1}$ \\ Joan T. Richtsmeier ${ }^{1, *}$
}

${ }^{1}$ Department of Anthropology, Pennsylvania State University, University Park, PA 16802

${ }^{2}$ Department of Computer Science and Engineering, University of Notre Dame, Notre Dame, IN 46556

\&These authors contributed equally to this paper

* Susan M. Motch Perrine and Joan T. Richtsmeier

Corresponding authors Email: qzk2@psu.edu and 1 ta10@psu.edu

Author Contributions: Designed research: SMMP, JTR, MKP, ELD, HZ, DZC, KK. Performed research: SMMP, MKP, ELD, MK, HZ, DZC, KK, JTR. Image segmentation: SMMP, MKP, JTR, HZ, DZC. Analyzed data: SMMP, MKP, ELD, HZ, KK, JTR. Contributed new analytical tools: HZ, DZC. Wrote the paper: SMMP, MKP, ELD, HZ, DZC, JTR. Edited the manuscript: SMMP, MKP, ELD, MK, HZ, DZC, KK, JTR.

Competing Interest Statement: The authors declare they have no relevant financial or non-financial competing interests to report.

Classification: Biological Sciences, Evolution

Keywords: craniofacial development, skull, embryonic cartilage, Crouzon syndrome, FGFR 


\begin{abstract}
The cranial endo- and dermal skeletons, which comprise the vertebrate skull, evolved independently and form separately during embryogenesis. In mammals, the mostly cartilaginous cranial endoskeleton forms prior to the bony dermatocranium. Many features of the chondrocranium are transient, undergoing endochondral ossification or disappearing, so its role in skull morphogenesis is not understood The fibroblast growth factor (FGF) and receptor (FGFR) signaling pathway contributes significantly to the regulation of osteochondroprogenitor cell function. Mutations in FGFR genes are associated with diseases that impact the skull including dwarfing chondrodyplasia and craniosynostosis syndromes. We investigate the developing chondrocranium and dermatocranium using a mouse model for craniosynostosis carrying a gain of function mutation in Fgfr2 to assess development of these cranial skeleton systems. Dermatocrania and chondrocrania of $\mathrm{Fgfr}_{2} \mathrm{C}^{\mathrm{C} 342 \mathrm{Y} /+}$ mice and their $\mathrm{Fgfr}_{2 \mathrm{c}} \mathrm{c}^{+/+}$littermates were quantified in 3D from microcomputed tomography images of mouse embryos. Chondrocrania of embryonic mice carrying the Fgfr2 mutation are larger than their $\mathrm{Fgfr}_{2} \mathrm{C}^{+/+}$littermates and include novel extensions of cartilage over the lateral and dorsal aspect of the brain. Like the forming chondrocranium, the embryonic dermatocranium is larger in Fgfr2 $\mathrm{c}^{\mathrm{C} 342 \mathrm{Y} /+}$ mice throughout embryogenesis but after disappearance of much of the chondrocranium, the dermatocranium becomes progressively smaller relative to Fgfr $2 \mathrm{c}^{+/+}$littermates during postnatal growth. Results reveal the direct effects of this Fgfr2c mutation on embryonic cranial cartilage, the impact of chondrocranial structure on developing dermatocranial elements, the importance of the chondrocranium in decoding the impact of specific genetic variants on head morphogenesis, and the potential for harnessing these effects as therapeutic targets.
\end{abstract}




\section{Significance Statement 120 words}

We present the first fully complete three-dimensional (3D) reconstructions of the mouse embryonic chondrocranium using a novel methodology of uncertainty guided segmentation of microcomputed tomography images with sparse annotation. We provide 3D reconstructions of chondrocrania of the Fgfr $2 c^{\mathrm{C} 342 \mathrm{Y} /+}$ Crouzon syndrome mouse and typically developing littermates for embryonic days 13.5 , $14.5,15.5,16.5$, and 17.5. This is the first study of the effects of an FGFR2 mutation on embryonic chondrocranial cartilage. 3D reconstructions of embryonic dermal bones reveal that the dermatocranium develops outside of, and in shapes that conform to the chondrocranium. Our findings have implications for the study and treatment of human craniofacial disease and for understanding the impact of chondrocranial morphology on the evolution of skull morphology.

\section{Main text}

\section{Introduction}

The heads of modern vertebrates have undergone drastic evolutionary transformations from the protective, predominantly cartilaginous assembly that surrounded the major cranial organs of early vertebrates. The emergence of the cranial endoskeleton was followed by the appearance of the cranial dermal skeleton and the cooperation of these two skeletal systems is a feature of vertebrate evolution. Most modern vertebrate skulls are integrated composites formed by the union of the endo- and dermal (exo-) cranial skeletons that form embryonically in cartilage and bone, respectively, and evolved as distinct systems (1). The cranial endoskeleton includes the cartilaginous chondrocranium and pharyngeal skeleton that form prior to cranial dermal bone formation in Osteichthyes (2-5). Elements of the mouse chondrocranium form individually in sequence beginning at embryonic day 12.5 (E12.5), fuse to provide an intricate protective covering for the brain and other sense organs, and parts of these elements begin to dissolve by E15.5 (4). That no modern vertebrate species has lost the chondrocranium during evolution suggests its essential role in skull development (2), and variation in chondrocranial anatomy across species (5) suggests its contribution to phylogenetic differences in skull morphology. Dermal bones of the skull arise individually in association with chondrocranial cartilages $(2,4)$ but are ultimately linked with other bones by sutures. Sutures are joints that facilitate circumferential growth of the skull through the coupled functions of suture mesenchyme that keeps adjacent bone separate, differentiating osteoblasts occupying a zone between the mesenchyme and osteogenic bony fronts, and addition of unmineralized 
matrix along the osteogenic fronts of individual bones (6-9). In craniosynostosis, osteoblasts that mineralize the suture before the completion of brain growth alter subsequent skull growth patterns and produce abnormal head shapes (10). In more severe craniosynostosis, skull deformity is coupled with increased intracranial pressure affecting brain health and neurocognitive function (11).

Approximately 1 in 2,000-2,500 children of all ethnic groups are born with craniosynostosis conditions $(12,13)$ and though variants of many genes are associated with these disorders $(8,13-20)$, alteration to the function of fibroblast growth factor receptor 2 (FGFR2) results in the more common craniosynostosis syndromes of Apert, Crouzon, and Pfeiffer. The Fgfr2c ${ }^{C 342 Y /+}$ Crouzon syndrome mouse model carries a cysteine to tyrosine substitution at amino acid 342 (Cys342Tyr; C342Y) in Fgfr2 equivalent to the FGFR2 mutation most often associated with Crouzon syndrome. The FGFR2c C342Y mutation, common to Pfeiffer and Crouzon syndromes (21-24), is associated with constitutive activation of the receptor, up-regulation of osteogenic differentiation $(13,21)$, and premature fusion of the coronal suture, typically prenatally. Research with mice has revealed that Fgfr2c is required for normal function of osteoblast lineage cells and interacts with Fgfr3, important to cells in the chondrocyte lineage during endochondral osteogenesis $(21,25)$.

The established explanation for cranial dysmorphogenesis in craniosynostosis syndromes -that premature closure of sutures results in changes in the growing skull (26)- assumes that suture closure is the primary insult, initiating changes in growth patterns and increased intracranial pressure that harm the brain and other cranial organs. Under this hypothesis, research into mechanism underlying craniosynostosis has focused primarily on how changes in genetic regulation affect osteoblast function, dermal bone formation, and mineralization of cranial suture mesenchyme, while typical therapies involve corrective and/or reconstructive surgery to adjust the size, shape, and position of skull bones to improve appearance and function. The recent definition of sutures as a mesenchymal stem cell niche $(27,28)$ provides a potential alternative approach to correcting closed sutures by combining biodegradable materials with mesenchymal stem cells to regenerate functional cranial sutures (11). But the composite observations that: skulls of mice carrying Fgfr2 mutations are dysmorphic prior to suture closure (29), tissues other than bone are dysmorphic in these mice at birth $(30,31)$, and that single cell transcriptome analysis of the murine embryonic coronal suture reveals a diversity of cell types within the suture $(7,8)$ 
suggest the potential importance of aberrant FGF/FGFR signaling on a variety of cranial cells and tissues. The development of a prenatal therapeutic approach requires a thorough understanding of cranial morphogenesis prior to the closure of sutures, knowledge that is currently incomplete.

The unique capacity of cartilage to grow interstitially enabling rapid, continuous growth in size and change in shape ensures customized protection for growing cranial organs prior to bone formation but also suggests the potential for the chondrocranium to influence the position and shape of dermal elements. We assess the effects of deviations in FGF/FGFR signaling on embryonic development of the chondrocranium and dermatocranium in Fgfr2 $\mathrm{C}^{\mathrm{C} 342 \mathrm{Y} /+}$ mice whose skull phenotype parallels that of humans with Crouzon/Pfeiffer syndrome $(21,30,32,33)$. The impact of this research is twofold: 1$)$ the samples and novel methods for embryonic cartilage visualization (34) and deep learning based segmentation using uncertainty-guided self-training with very sparse annotation (35) allow us to address questions inaccessible in the study of humans but inform us about human craniofacial development and disease process; and 2) our 3D morphological analyses provide a unique opportunity for innovative evaluation of the effect of a mutation on embryonic cranial cartilage formation and on the relationship between chondrocranial cartilage and dermal bone formation. We anticipate one of three outcomes: i) chondrocranial morphology of Fgfr2 $\mathrm{C}^{\mathrm{C} 342 \mathrm{Y} /+}$ mice and that of their controls, Fgfr2c $\mathrm{C}^{+/+}$(unaffected) littermates, are similar indicating that the mutation affects the cranial osteoblast lineage but not the chondrocyte series; ii) chondrocranial morphology separates Fgfr $2 c^{\mathrm{C} 342 Y /+}$ mice from Fgfr $2 \mathrm{c}^{+/+}$littermates indicating that the mutation affects the chondrocyte series and the osteoblast lineage but the morphological effects of the mutation on the dermatocranium and the chondrocranium are dissociated; or iii) chondrocranial morphology separates Fgfr2 $\mathrm{C}^{\mathrm{C} 342 \mathrm{Y} /+}$ mice from $\mathrm{Fgfr}_{2 \mathrm{C}}{ }^{+/+}$littermates and the morphological effects of the mutation on chondrocranial cartilages and dermatocranial bone show correspondence, emphasizing integration of chondrocranial and dermatocranial development. We provide a hypothesis of the role of the chondrocranium in dermatocranium development in craniosynostosis and by extension, in normal development, and in evolution.

\section{Results}

Segmentation and visualization of embryonic mouse cranial bone and cartilage in 3D. Embryonic bone was segmented from microCT images by thresholding techniques using Avizo 2020.2 (Thermo 
Fisher Scientific; see supplemental materials), but segmenting embryonic cranial cartilage using deep learning based, fully convolutional networks (FCNs) (36-38) remains a challenging task. The difficulty involves a combined cadre of conditions including significant topological variation across cartilages, largesize image volumes $(\bar{X} \approx 1300 \times 1700 \times 2000$ voxels), extremely thin regions-of-interest (ROIs), and unavailability of voxel-wise annotation of whole volumes for network training. Our goal was to enable automated segmentation over developmental time but full annotation (i.e., labeling all ROls in a sufficient number of whole 3D volumes) for training deep learning based, FCN (36-38) models for chondrocranium segmentation is impractical. The reasons include large image size necessary to capture biological complexity, substantial changes in corresponding anatomical regions across developmental time and genotypes, and the need for sample sizes adequate to achieve statistical power. Consequently, a new two-phase approach implementing sparse annotation was used for training our segmentation model. The two-phase approach involves automatically segmenting the chondrocranium with very sparse annotation to bridge the performance gap relative to full annotation and integrating limited human corrections to finetune the model. Our two-phase approach (supplemental materials) built on an automatic segmentation procedure (35) and produced fully 3D reconstructions of the chondrocranium at each embryonic age for Fgfr2 $\mathrm{c}^{\mathrm{C} 342 \mathrm{Y} /+}$ mice and their $\mathrm{Fgfr} 2 \mathrm{c}^{+/+}$littermates, which were first evaluated for cartilage thickness using Avizo 2020.2 (Fig 1). 
A

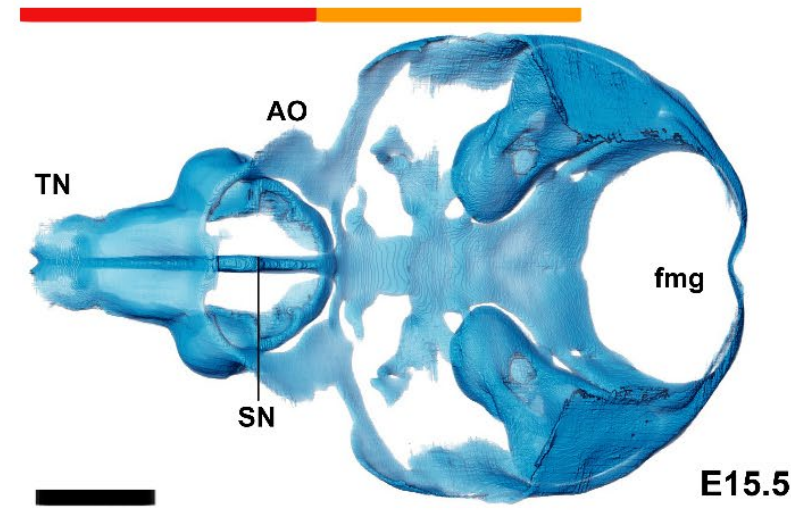

B

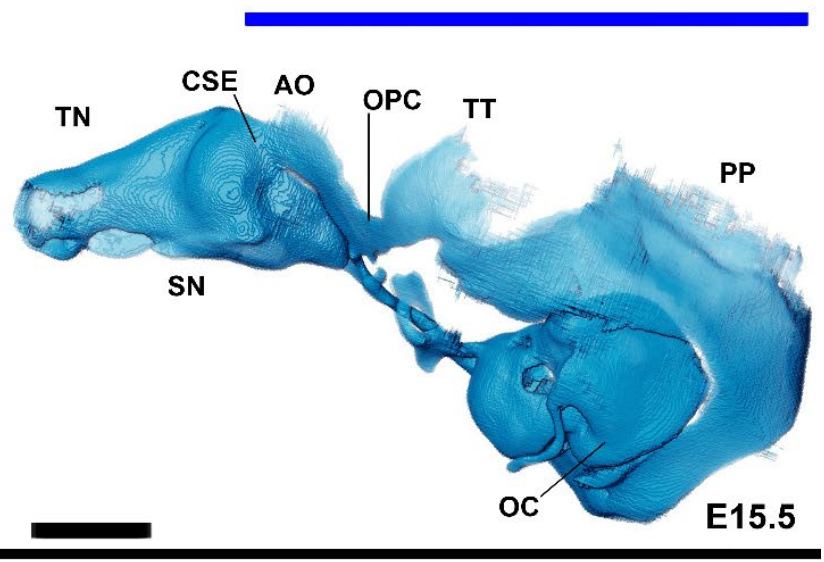

E15.5

E16.5

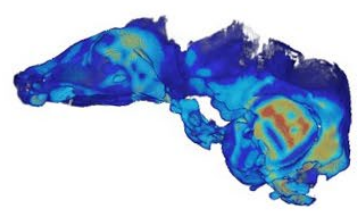

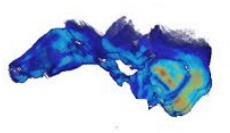
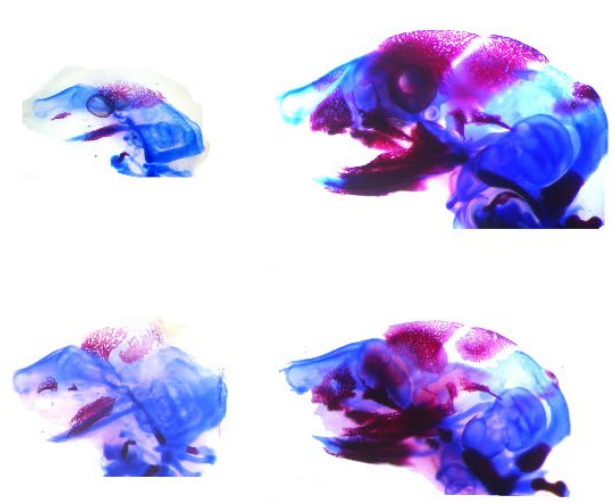

F Fgfr2 $\mathrm{c}^{+/+}$

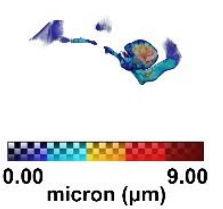

\section{E Fgfr2c $\mathrm{F}^{+/ t}$}
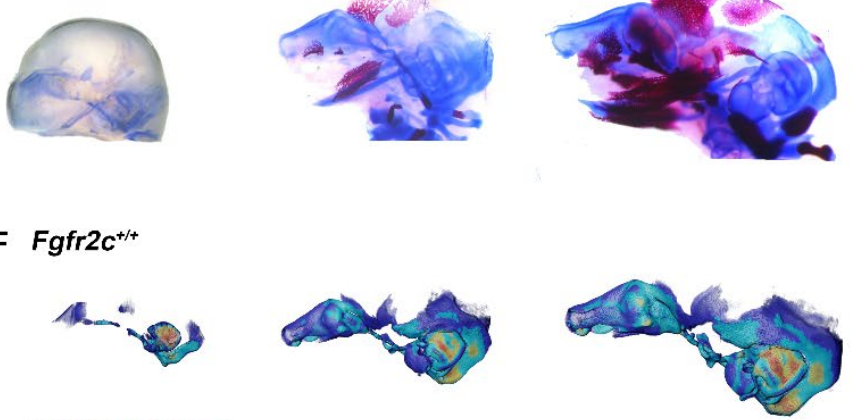
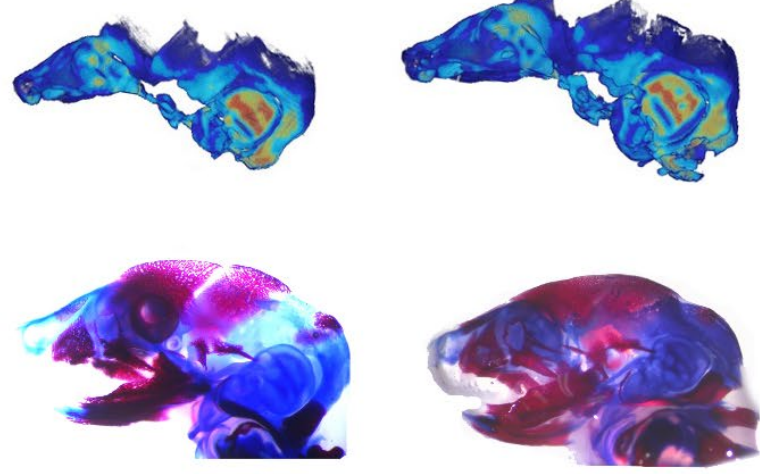
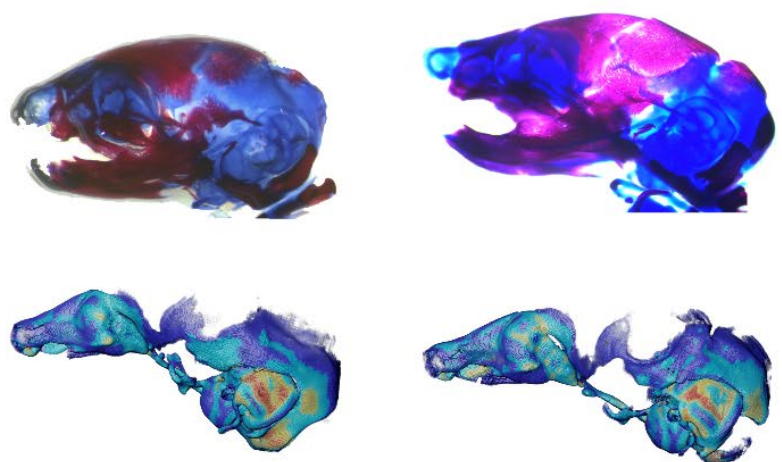
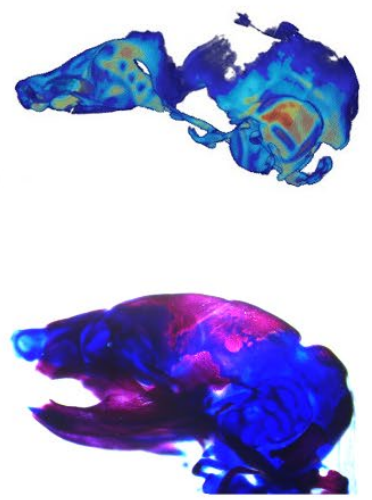

E17.5

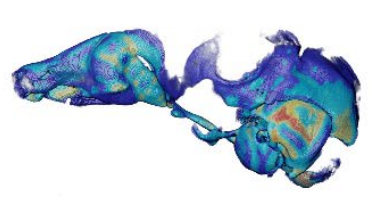

Figure 1. Morphology of the mouse embryonic chondrocranium and cranial skeleton. The $F g f r 2 \mathrm{C}^{+/+}$mouse chondrocranium at $\mathrm{E} 15.5$ ( $\mathrm{A}$, superior and $\mathrm{B}$, lateral views) is complete, consisting of the olfactory region ( $A$, red bar), braincase floor ( $A$, yellow bar), and lateral walls of the preoccipital and occipital regions (B, blue bar). Specific areas of interest include the ala orbitalis (AO), sphenethmoid commissure (CSE), otic capsule (OC), parietal plate (PP), septum nasi (SN), tectum nasi (TN), orbitoparietal commissure (OPC), and tectum transversum (TT) cartilages and the foramen magnum (fgm). Thickness maps of the chondrocranium of Fgfr2 $\mathrm{C}^{\mathrm{C} 342 \mathrm{Y} / \mathrm{+}}$ mice segmented from PTA-enhanced microCT images (C) at E13.5, E14.5, E15.5, E16.5, and E17.5 (from left to right), and similarly aged cleared and stained specimens (D). Cleared and stained $\mathrm{Fgfr}_{\mathrm{C}} \mathrm{C}^{+/+}$mice $(\mathrm{E})$ and thickness maps $(\mathrm{F})$ segmented from PTA-enhanced microCT 
images. Scalebars $=1 \mathrm{~mm}$. Colormap indicates cartilage thickness in $\mathrm{C}$ and $\mathrm{F}$, which ranged from just over $0 \mu \mathrm{m}$ (dark blue) to nearly $9 \mu \mathrm{m}$ (dark red).

\section{Morphology of the mouse embryonic chondrocranium E13.5-E17.5.}

The appearance of the parachordal cartilages marks the initiation of the chondrocranium in C57BL/6J mice at E12.5 (2, 3) with ongoing appearance and growth of chondrocranial cartilages (Fig. 1) (2, 4). By E13.5, the lateral wall of the preoccipital region of Fgfr $2 c^{C 342 Y /+}$ mice consists of well-developed ala orbitalis (AO), sphenethmoid commissure (CSE), and tectum transversum (TT), while $F g f r 2 \mathrm{C}^{+/+}$mice do not develop these structures until E14.5. The AO and TT extend further apically over the developing brain and are larger in Fgfr2 $\mathrm{c}^{\mathrm{C} 342 \mathrm{Y} / \mathrm{+}}$ mice, and the orbitoparietal commissure (OPC) that connects the TT to the parietal plate (PP) at their bases contains more cartilage along its apical lip. This results in a broader and thicker rim of cartilage along the lateral wall, which in some Fgfr $2 c^{C 342 Y /+}$ individuals provides uninterrupted coverage of the lateral aspect of the preoccipital region (Fig. 1). The AO, TT, and OPC begin to disappear around E16.5 in both genotypes, becoming thinner and taking on a lace-like appearance, but remain more complete in $\mathrm{Fgfr}_{2} \mathrm{c}^{\mathrm{C} 342 \mathrm{Y} /+}$ mice relative to $\mathrm{Fgfr} \mathrm{c}^{+/+}$mice at E17.5. Additional parts of the chondrocranium begin to thin and disappear in both genotypes as the dermatocranium thickens and expands after E17.5.

We used a suite of landmarks whose 3D coordinates could be reliably located across embryonic age groups (Table S1 and Table S2) to estimate differences in chondrocranial morphology. To test for differences in regional shape between $\mathrm{Fgfr} 2 \mathrm{c}^{\mathrm{C} 342 \mathrm{Y} /+}$ and $\mathrm{Fgfr}_{2} \mathrm{C}^{+/+}$chondrocrania, we analyzed three distinct configurations of 3D landmark coordinates representing cartilages of the nasal capsule, of the braincase floor, and of the lateral walls and roof of the vault using Euclidean Distance Matrix Analysis (EDMA) (39) to statistically test for differences in shape (see Experimental Procedures section). Confidence interval testing for individual linear distances identifies localized differences between genotypes. Details on sample size (Table S3), landmarks used (Table S1 and Table S2), and results of statistical tests of the null hypothesis of similarity in shape for each region (Table S4) are included in Supporting Information (SI).

At E13.5, delayed development of some structures made acquisition of all landmarks impossible and sample sizes were small $(\mathrm{N}=3)$, so statistical testing was inconclusive. Still, $77 \%$ of all linear 
bioRxiv preprint doi: https://doi.org/10.1101/2021.11.24.469914 this version posted November 25,2021 . The copyright holder for this preprint

(which was not certified by peer review) is the author/funder, who has granted bioRxiv a license to display the preprint in perpetuity. It is made available under aCC-BY-NC-ND 4.0 International license.

distances were larger in Fgfr2c ${ }^{C 342 Y /+}$ chondrocrania at E13.5, and of those, 40\% showed increased size in Fgfr2 $c^{\mathrm{C} 342 Y /+}$ mice ranging from $5 \%$ to $46 \%$. By E14.5, over half of the linear distances among chondrocranial landmarks are $5-30 \%$ larger in $F g f r 2 c^{C 342 Y /+}$ mice indicating a sustained, global increase in size relative to $\mathrm{Fgfr}_{2 \mathrm{C}}^{+/+}$littermates, though not all differences are statistically significant (see SI). At E14.5 and E15.5, measures that summarize the entire chondrocranium are larger in Fgfr2 $\mathrm{c}^{\mathrm{C} 342 \mathrm{Y} /{ }^{+}}$mice. This difference becomes more localized with development so that by E17.5, significant differences are concentrated in the lateral walls of the preoccipital region up to the posterior aspect of olfactory capsule. 
bioRxiv preprint doi: https://doi.org/10.1101/2021.11.24.469914; this version posted November 25,2021 . The copyright holder for this preprint (which was not certified by peer review) is the author/funder, who has granted bioRxiv a license to display the preprint in perpetuity. It is made available under aCC-BY-NC-ND 4.0 International license.

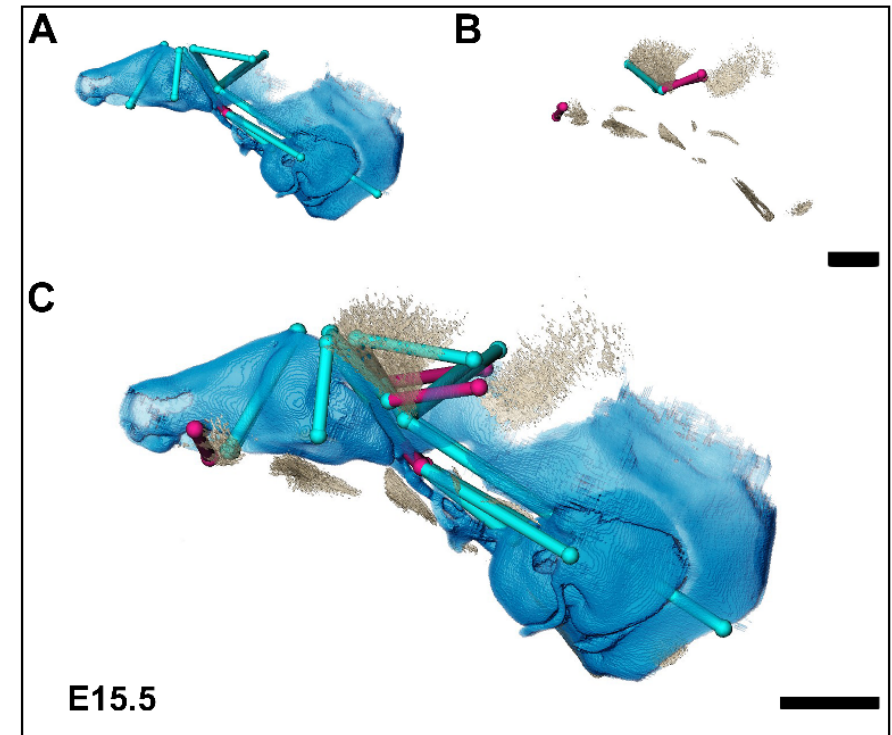

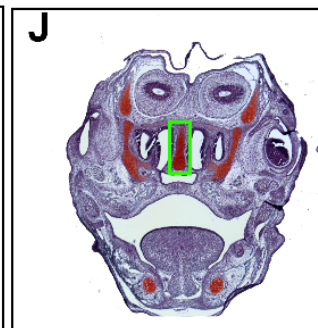

Nasal Septum
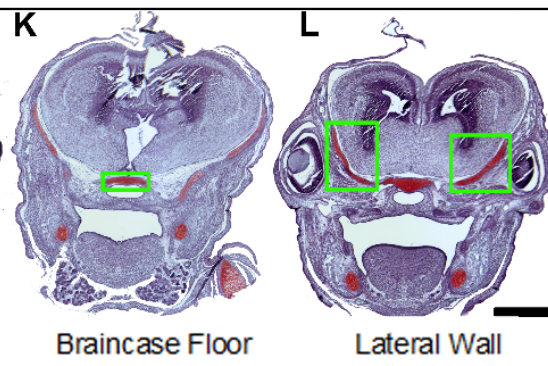

M

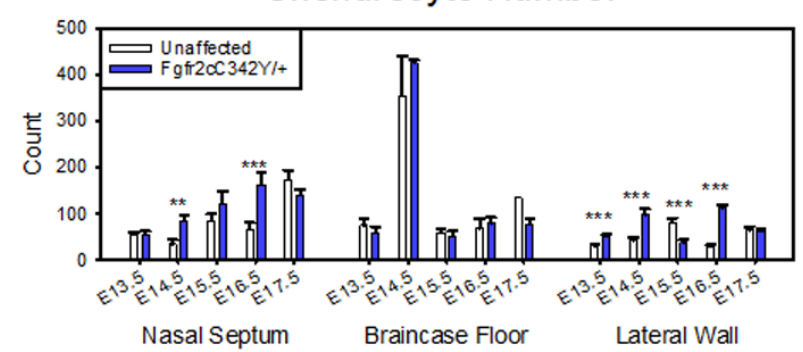

$\mathbf{N}$

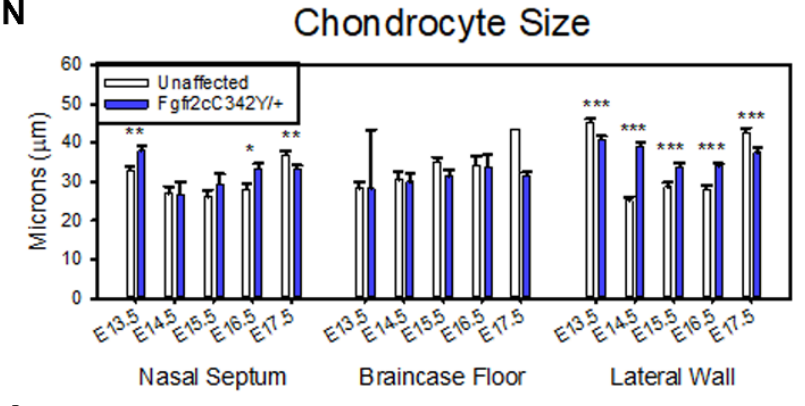

0

Matrix Area

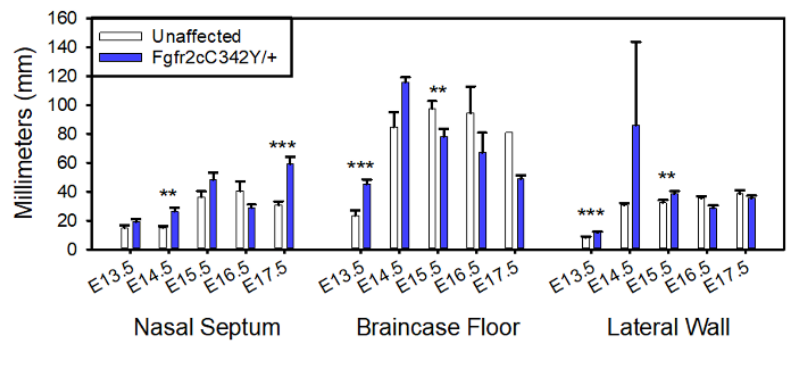

E17.5

E

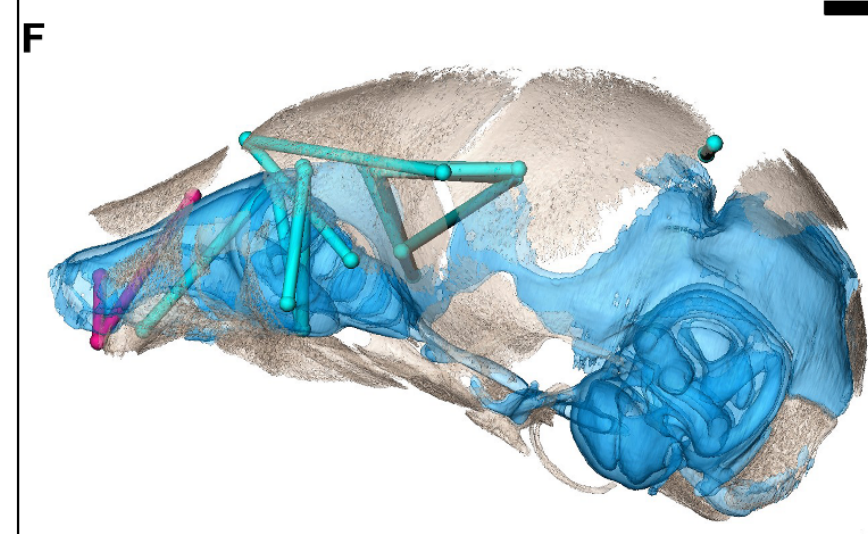

-

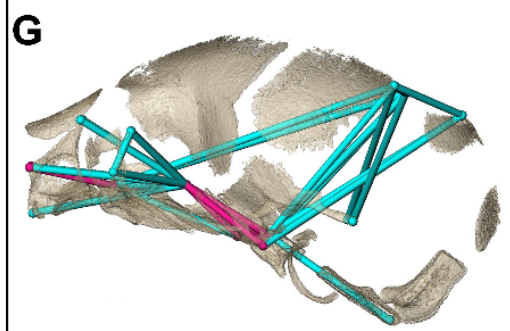

E17.5

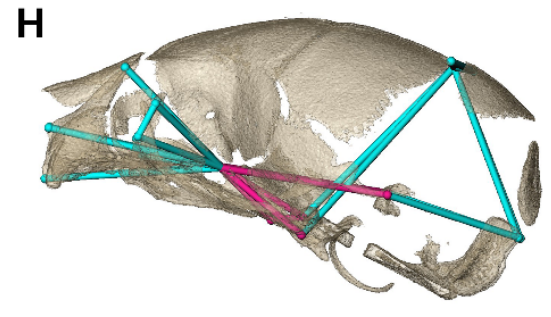

Po

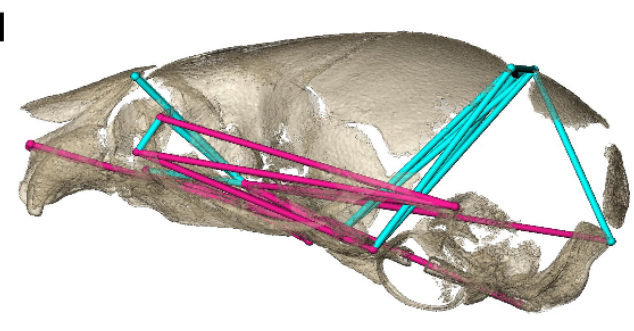

P2 
Figure 2. EDMA of the chondrocranium and bony skull and histomorphology of the chondrocranium. Linear distances of the chondrocranium $(A, D)$, bony skull $(B, E)$ and the two superimposed $(C, F)$ that are statistically significantly different between genotypes by confidence interval testing $(\alpha=0.10)$ are shown. Blue lines indicate linear distances that are significantly larger in Fgfr2 $c^{C 342 Y /+}$ mice; fuchsia lines are significantly reduced in Fgfr2 $c^{C 342 Y /+}$ mice. A limited landmark set common to the chondrocranium and bony skull was used for E15.5 (A-C) and E17.5 (D-F) analyses and indicated that the lateral wall and olfactory regions are most different between Fgfr2 $\mathrm{C}^{\mathrm{C} 342 \mathrm{Y} /+}$ and $\mathrm{Fgfr} 2 \mathrm{C}^{+/+}$mice at these ages. Increased mineralization allowed a larger set of landmarks to be used for statistical comparison of skull shape between genotypes at E17.5 $(\mathrm{G}), \mathrm{P0}(\mathrm{H})$, and $\mathrm{P} 2(\mathrm{I})$ revealing a reversal in the relative size of the Fgfr2 $\mathrm{C}^{+/ C 342 Y}$ skull postnatally (I). E15.5 septum nasi (J), braincase floor $(\mathrm{K})$, and lateral walls $(\mathrm{L})$ of the chondrocranium are shown. These areas were assessed at E13.5, E14.5, E15.5, E16.5, and E17.5 for chondrocyte number $(\mathrm{M})$, chondrocyte size $(\mathrm{N})$, and area of cartilaginous matrix $(\mathrm{O})$ in $\mathrm{Fgfr} 2 \mathrm{c}^{\mathrm{C} 342 \mathrm{Y} / \mathrm{+}}$ and Fgfr $2 \mathrm{C}^{+/+}$mice. In agreement with the larger chondrocranium in Fgfr $2 \mathrm{c}^{\mathrm{C} 342 \mathrm{Y} /+}$ mice, there are localized regions that reveal increases in chondrocyte number, size, and/or contribution of matrix at each timepoint. Note the trend of increasing numbers of chondrocytes over time as expected in a growing chondrocranium (M). For histological analysis ${ }^{*} p \leq 0.05,{ }^{* *} p \leq 0.01,{ }^{* * *} p \leq 0.001$. Scalebars $=1 \mathrm{~mm}$.

For all ages considered, linear distances that measure the width and rostrocaudal length of the walls of the pre- and post-occipital region are larger in $\mathrm{Fgfr}_{2 \mathrm{C}} \mathrm{C342 \textrm {Y } / +}$ mice relative to $\mathrm{Fgfr} \mathrm{C}^{+/+}$littermates (Fig 2A, D). The apparent reduction in the height of the TT in Fgfr2C $\mathrm{C}^{\mathrm{C} 42 \mathrm{Y} /+}$ mice (Fig 2A, D) is due to excess cartilage deposited along the apical edge of the OPC in Fgfr2 $\mathrm{c}^{\mathrm{C} 342 \mathrm{Y} /+}$ mice reducing the distance between the OPC and TT. Neither the braincase floor nor the olfactory region shows consistent differences across embryonic ages but instead shows a high degree of variation across both genotypes at these ages. The cartilages of the braincase floor are similar in shape between groups at E14.5 and E15.5 but dimensions are relatively larger in Fgfr2 $\mathrm{c}^{\mathrm{C} 342 \mathrm{Y} /+}$ mice. The magnitude of the observed differences of braincase floor dimensions diminishes with age and there are no statistically significant differences by E17.5. The olfactory region is significantly larger in all dimensions in Fgfr2 $c^{\mathrm{C} 342 Y /+}$ mice at E14.5, with some dimensions being as much as $25 \%$ larger relative to $\mathrm{Fgfr}_{2 \mathrm{C}}{ }^{+/+}$littermates. By E17.5, the relative increase in the olfactory region is reduced to a range of 5 to $15 \%$.

\section{Cellular characterization of embryonic cartilage of the chondrocranium.}

Observations of growth plate cartilages identify cellular processes that contribute to cartilage growth as chondrocyte proliferation, hypertrophy, and matrix deposition (40-42). To investigate the cellular basis of morphological differences in chondrocranial morphology we analyzed the number and size of chondrocytes and the amount of matrix per region of interest in the septum nasi, braincase floor, and the lateral walls of the chondrocranium in Fgfr2 $\mathrm{c}^{\mathrm{C} 342 \mathrm{Y} /+}$ mice relative to $\mathrm{Fgfr}^{+\mathrm{c}^{+/+}}$littermates at E13.5, E14.5, E15.5, E16.5, and E17.5 (Fig. 2J-O). 
Our analysis focused on the greater part of the nasal septum that remains as cartilage in the adult. There were significantly more chondrocytes in Fgfr2 $c^{C 342 Y /+}$ nasal septa at $E 14.5(p=0.006)$ and E16.5 ( $p \leq 0.001)$ relative to Fgfr2 $c^{+/+}$littermates (Fig. 2M). Chondrocytes in the nasal septum were larger in Fgfr2 $c^{\mathrm{C} 342 Y /+}$ mice at E13.5 $(\mathrm{p}=0.004)$ and E16.5 $(\mathrm{p}=0.016)$ but at E17.5, chondrocytes of $\mathrm{Fgfr}^{\mathrm{c}} \mathrm{c}^{+/+}$ littermates were larger $(p=0.008)(F i g .2 N)$. The amount of matrix within the nasal septum was increased at E14.5 ( $p=0.003)$ and E17.5 ( $\mathrm{p} \leq 0.001)$ in Fgfr2 $\mathrm{c}^{\mathrm{C} 342 \mathrm{Y} /+}$ mice relative to Fgfr2 $\mathrm{c}^{+/+}$littermates (Fig. 2O).

Histological analysis of braincase floor cartilage that mineralizes endochondrally indicates no changes in chondrocyte number or size between genotypes at any of the ages investigated, in agreement with our observation of similarity of 3D morphology of the braincase floor cartilages. The amount of matrix was increased in the braincase floor cartilage of $F g f r 2 c^{C 342 Y /+}$ mice at $E 13.5(p \leq 0.001)$ but this relationship was reversed at $\mathrm{E} 15.5$ ( $p=0.013)$ (Fig. 2O).

We identified many differences in the cartilages of the lateral walls of the chondrocranium that contribute to morphological differences between genotypes and reflect the early deposition of cartilage and disintegration of lateral wall cartilages at E16.5 associated with dermal bone mineralization (2). More chondrocytes were identified in lateral wall cartilages of Fgfr2 $c^{C 342 Y /+}$ mice at E13.5 ( $\left.p \leq 0.001\right), \mathrm{E} 14.5$ $(p \leq 0.001)$, and E16.5 (p $\leq 0.001)$, but at E15.5 there are more cells in the lateral walls of $F g f r 2 c^{+/+}$ individuals $(p \leq 0.001)$ (Fig. 2M). Cell size is greater in Fgfr2 $c^{+/+}$littermates during early $(E 13.5, p \leq 0.001)$ and late $(E 17.5, p \leq 0.001)$ prenatal development. In between these timepoints chondrocytes are larger in Fgfr2 $c^{C 342 Y /+}$ mice $(E 14.5 p \leq 0.001, E 15.5 p=0.001, E 16.5 p \leq 0.001)$ which is consistent with the identification of a larger chondrocranium in the Fgfr2c $\mathrm{C}^{\mathrm{C} 34 \mathrm{Y} /+}$ mice (Fig. $2 \mathrm{~N}$ ). Area of the cartilage matrix is greater in Fgfr2 $c^{C 342 Y /+}$ mice at E13.5 $(p \leq 0.001)$ and E15.5 $(p=0.010)$. The increase in chondrocytes in $\mathrm{Fgfr}_{2 \mathrm{C}} \mathrm{C}^{+/+}$individuals at E15.5 is followed by an increase in cartilage matrix area in $\mathrm{Fgfr}_{2} \mathrm{c}^{+/+}$individuals at E16.5 ( $p \leq 0.001$ ) (Fig. 2O). Consequently, the significantly larger chondrocytes in Fgfr2c ${ }^{C 342 Y /+}$ mice at E16.5 account for the larger lateral wall cartilages observed. We generally observed a trend of more chondrocytes, larger chondrocytes, and/or more matrix in the Fgfr2 $\mathrm{c}^{\mathrm{C} 342 \mathrm{Y} /+}$ mice as compared to their $\mathrm{Fgfr}_{2 \mathrm{C}} \mathrm{C}^{++}$littermates at all timepoints prior to the disintegration of the chondrocranium beginning at E16.5.

\section{The bony skull.}

\section{Coronal suture fusion and bone volume.}


Initial mineralization of cranial dermal bone is apparent by alizarin red staining at E14.5 (Fig. 1D,E), but individual cranial bones are not easily detected by microCT until E15.5 (Fig. 2B). Using microCT, none of the mice show fusion of the coronal suture prior to birth but half of $F g f r 2 c^{C 342 Y /+}$ mice show bridging of one or both coronal sutures at E17.5 (9/18), and by birth or postnatal day 0 (P0), all Fgfr2c ${ }^{\mathrm{C} 342 \mathrm{Y} /+}$ mice $(11 / 11)$ show partial or complete closure of one or both coronal sutures. Coupled with evidence by alizarin red staining of partially fused sutures at E17.5 by other investigators (43) this confirms that coronal suture closure occurs between E17.5 and P0 in most Fgfr2 $c^{\mathrm{C} 342 \mathrm{Y} /+}$ mice (30) (see Supporting Information Fig 2 for coronal and other suture closure patterns).

Bone size and volume are highly variable in both genotypes during prenatal development, but estimation of individual bone volumes reveals that some dermal bones (i.e., nasal, palatine, vomer) and an endochondral bone (basioccipital) are significantly larger in $\mathrm{Fgfr} 2 \mathrm{c}^{\mathrm{C} 342 \mathrm{Y} /+}$ mice at $\mathrm{P} 0$ relative to $\mathrm{Fgfr} 2 \mathrm{c}^{+/+}$ littermates. However, at E17.5, only the vomer is significantly larger in Fgfr2 $\mathrm{c}^{\mathrm{C} 342 \mathrm{Y} /{ }^{+}}$mice relative to Fgfr2 $\mathrm{c}^{+/+}$littermates (Table S6).

\section{Morphometric comparison of pre- and post-natal Fgfr2 $c^{\mathrm{C342Y/+}}$ Crouzon mouse bony skull.}

Skulls of adult Fgfr2 $c^{\mathrm{C} 342 \mathrm{Y} /+}$ mice show closure of the coronal sutures and small size (21), with a domed cranial vault and skull lengths reduced by as much as $20 \%$ (33). We used EDMA (34) and three distinct configurations of 3D landmark coordinates representing bones of the facial skeleton, braincase floor, and lateral walls and roof of the vault whose 3D coordinates could be reliably located across age groups (Table S2) to estimate differences in bony skull morphology. Contrary to what is known of postnatal Fgfr2 $c^{C 342 Y /+}$ skulls, our analyses reveal that Fgfr2 $c^{C 342 Y /+}$ embryonic and early postnatal skulls are larger than those of $\mathrm{Fgfr} \mathrm{C}^{+/+}$littermates $(30,31)$ (Table S5). Details on sample size, landmarks used, and statistical results are included in supplemental tables and figures (Tables S1-S5).

The lateral wall and roof of the cranial vault consist of dermal bones that show marked variability within and between genotypes at E15.5 most likely due to differences in developmental timing (44). Dimensions of the frontal and parietal bone are significantly larger in Fgfr2 $\mathrm{C}^{\mathrm{C} 342 \mathrm{Y} / \mathrm{+}}$ mice at this age, some by as much as $15-30 \%$. Nearly all dimensions of the lateral walls and roof of the vault are larger in Fgfr2 $c^{\mathrm{C} 342 \mathrm{Y} /+}$ mice at E16.5, E17.5, and P0 indicating a pattern of increased dermal bone growth in mice carrying the Fgfr2c $\mathrm{C} 342 \mathrm{Y}$ mutation. At P0, this pattern begins to change and by $\mathrm{P} 2$ all measures 
oriented along the rostral-caudal axis are relatively reduced in Fgfr2 $\mathrm{c}^{\mathrm{C} 342 \mathrm{Y} /+}$ mice, but measures of width of the lateral walls at the level of the coronal suture and of the occipital walls remain relatively large in Fgfr2 $c^{C 342 Y /+}$ mice at $\mathrm{P} 2$. There are no differences in braincase floor morphology between genotypes at E15.5 but as development proceeds, the braincase floor of Fgfr2 $\mathrm{C}^{\mathrm{C} 342 \mathrm{Y} /+}$ mice becomes larger across all dimensions relative to $\mathrm{Fgfr}^{+} \mathrm{C}^{+/+}$littermates. By P2, only the most posterior aspect of the braincase floor remains relatively large in Fgfr2c ${ }^{C 342 Y /+}$ mice. Bones of the Fgfr2 $c^{C 342 Y /+}$ facial skeleton show relatively few significant differences prenatally but by P0 the facial skeleton of $F g f r 2 c^{C 342 Y /+}$ mice is significantly larger than that of $\mathrm{Fgfr}_{2} \mathrm{c}^{+/+}$littermates. Dimensions of the Fgfr $2 \mathrm{c}^{\mathrm{C} 342 \mathrm{Y} /+}$ facial skeleton remain wide relative to $\mathrm{Fgfr}_{2 \mathrm{C}^{+/+}}$littermates at $\mathrm{P} 2$ but are reduced rostro caudally relative to $\mathrm{Fgfr} 2 \mathrm{c}^{+/+}$littermates at $\mathrm{P} 2$.

The increasing amount of mineralized bone with age enabled use of a larger number of landmarks $(K=24)$ for a comparative analysis of late embryonic $(E 17.5)$, newborn $(P 0)$ and early postnatal (P2) skull morphology (see Table S2) (Fig. 2G, H, I). At E17.5, as the lateral walls of the chondrocranium dissolve but prior to coronal suture fusion, global shape is statistically significantly different between genotypes and confidence interval testing reveals a generally larger facial skeleton surrounding the olfactory capsule, shortened and narrowed anterior braincase floor, and an expanded posterior cranial vault in Fgfr2 $c^{\mathrm{C} 342 \mathrm{Y} /+}$ mice (Fig. $2 \mathrm{G}$ ). This general pattern continues at $\mathrm{P} 0$ though the magnitude of the differences is reduced (Fig. $2 \mathrm{H}$ ). By P2, the posterior cranial vault remains larger than normal, but many dimensions of the facial skeleton and braincase floor are now small relative to $F g f r 2 c^{+/+}$littermates suggesting that these differences are a result of altered postnatal growth patterns in $\mathrm{Fgfr} 2 \mathrm{c}^{\mathrm{C} 342 \mathrm{Y} /+}$ mice (Fig. 2l).

\section{Morphological Integration.}

Morphological integration (MI) refers to patterns of correlation and/or covariation among organismal traits with the degree of integration measured by the intensity of statistical association in the phenotype. Patterns of covariation emerge because organisms are constructed of units, or modules, that are coherent within themselves yet part of a larger unit. Modules result from structural or developmental associations within an organism $(31,45,46)$, but can also be outcomes of sample-specific developmental architecture and variation (47) indicative of shared regulatory processes $(48,49)$. We use a comparative study of $\mathrm{MI}$ of the chondrocranium and dermatocranium in $\mathrm{Fgfr} 2 \mathrm{c}^{\mathrm{C} 342 \mathrm{Y} /+}$ mice and $\mathrm{Fgfr} \mathrm{c}^{+/+}$littermates to 
determine whether coordinated patterns of association within and between these modules are altered by a genetic variant.

Linear distances within the chondrocranium and dermatocranium were estimated from 3D coordinates of landmarks (see SI Table S1) and used to statistically compare MI patterns (50) in Fgfr2 $\mathrm{c}^{\mathrm{C} 342 \mathrm{Y} /+}$ and $\mathrm{Fgfr} 2 \mathrm{c}^{+/+}$mice within the chondrocranium, within the dermatocranium, and between chondrocranium and dermatocranium at E15.5 and E17.5. We consider $\bar{r} \geq 0.60$ as indicative of a relatively strong association, whether the correlation is positive or negative.

At E15.5, the mean of the absolute values of the correlation coefficients among chondrocranial dimensions is increased in Fgfr $2 c^{C 342 Y /+}$ mice $(\bar{r}=0.73)$ relative to $F g f r 2 c^{+/+}$mice $(\bar{r}=0.53)$ but the pattern of correlation is similar in the two samples with few (14\%) correlations significantly different between the two genotypes (Table S7). By E17.5 the mean of the absolute values of the correlation coefficients have decreased in both samples but remain relatively high in Fgfr $2 c^{C 342 Y /+}$ mice $(\bar{r}=0.61)$ and the number of within-chondrocranial correlation coefficients that are significantly different between the samples is further

Table 1. Mean $(\bar{x})$ and standard deviation (s) of the absolute value of correlation coefficients for all chondrocranium measures, all dermatocranium measures, and between all chondrocranium and dermatocranium measures for all E15.5 and E17.5 samples used in analysis.

\begin{tabular}{|cc|c|c|c|c|c|c|}
\multicolumn{2}{c}{} & \multicolumn{2}{c}{ Dermatocranium (B) } & \multicolumn{2}{c}{ Chondrocranium (C) } & \multicolumn{2}{c|}{ D\&C } \\
\hline \multirow{2}{*}{ Age } & Genotype & $\bar{x}$ & $\mathrm{~s}$ & $\bar{x}$ & $\mathrm{~s}$ & $\bar{x}$ & $\mathrm{~s}$ \\
\hline \multirow{2}{*}{ E15.5 } & Affected & 0.62 & 0.33 & 0.73 & 0.25 & 0.65 & 0.30 \\
\cline { 3 - 8 } & Unaffected & 0.68 & 0.31 & 0.53 & 0.29 & 0.42 & 0.25 \\
\cline { 3 - 8 } E17.5 & Affected & 0.59 & 0.29 & 0.61 & 0.28 & 0.46 & 0.26 \\
\cline { 3 - 8 } & Unaffected & 0.52 & 0.28 & 0.47 & 0.28 & 0.49 & 0.27 \\
\hline
\end{tabular}

reduced $(9 \%)$. These results reveal a remarkable correspondence in overall patterns of within-

chondrocranial associations in the two genotypes and a marked increase in magnitude of correlations in Fgfr2c $\mathrm{C}^{\mathrm{C} 42 \mathrm{Y} /+}$ mice.

At E15.5, approximately one day after the initial mineralization of cranial dermal bone, the mean of the absolute values of correlation coefficients among dermatocranial dimensions are relatively strong in both genotypes (Table 1 ) and only twenty (9\%) of the correlation coefficients among dermatocranial dimensions are significantly different between the two genotypes. By E17.5 the mean of the absolute 
value of correlation coefficients have decreased in both samples, though decrease is less in Fgfr2c ${ }^{C 342 Y /+}$ mice, and a similarly small number of correlations are significantly different between genotypes.

At E15.5 association of chondrocranium and dermatocranium in the Fgfr2 $c^{C 342 Y /+}$ mice is strong $(\bar{r}$ $=0.65)$ relative to their $F g f r 2 c^{+/+}$littermates $(\bar{r}=0.42)$ and statistical analysis of the difference in MI reveals $183(41.5 \%)$ of the correlations to be significantly different between genotypes. Of these significant differences, $124(28.1 \%)$ are due to a greater absolute magnitude of the correlation in Fgfr2 $\mathrm{c}^{\mathrm{C} 342 \mathrm{Y} /+}$ mice relative to $\mathrm{Fgfr} \mathrm{c}^{+/+}$littermates while $59(13.4 \%)$ of the differences are due to a significantly stronger association among $\mathrm{Fgfr}_{2} \mathrm{c}^{+/+}$littermates. At E15.5, the significant differences in correlation patterns are of two general types:1) correlations between specific chondrocranium and dermatocranium measures are moderatly to strongly negative in $\mathrm{Fgfr}_{2} \mathrm{c}^{+/+}$littermates while being strongly positive in Fgfr2 $c^{\mathrm{C} 342 \mathrm{Y} /+}$ mice indicating pairs of measures that vary in opposite directions in typically developing mice but that tend to increase (or decrease) jointly when the Fgfr2 mutation is present ; and 2)
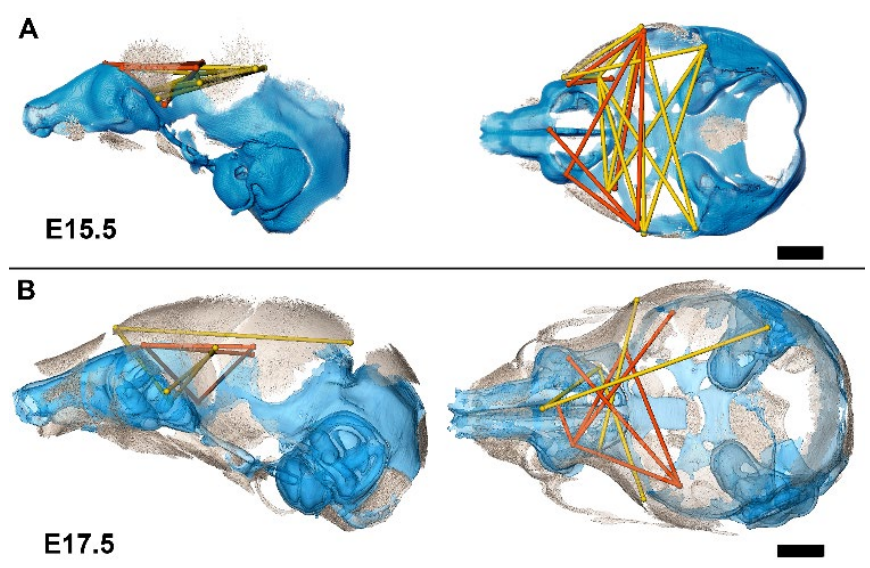

Figure 3. Summary of differences in morphological integration of dermatocranium and chodrocranium between genotypes. Pictured linear distance pairs from the dermatocranium (yellow) and chondrocranium (orange) whose association is statistically stronger $(\alpha=0.10)$ in Fgfr2 $c^{C 342 Y /+}$ mice relative to Fgfr2c $\mathrm{C}^{+/+}$mice at E15.5 (A) and E17.5 (B). Left lateral and superior views shown. Scalebars $=1$ $\mathrm{mm}$. correlations that are moderately positive in

Fgfr2 $\mathrm{C}^{+/+}$mice and strongly negative in

Fgfr $2 c^{C 342 Y /+}$ mice describing relationships among chondrocranial and dermatocranial measures that are of low to medium positive intensity in typically developing mice but that vary strongly in opposite directions when the Fgfr2 mutation is present (Fig 3A). By E17.5, the lateral walls of the chondrocranium are dissolving as the dermal bones expand and mineralize and the mean association between the two modules decreases in Fgfr2 $\mathrm{C}^{\mathrm{C} 342 \mathrm{Y} /{ }^{+}}$mice and increases slightly in $\mathrm{Fgfr}_{2 \mathrm{C}}^{+/+}$mice yielding similar mean values. The

number of significant differences in correlations between dermatocranial and chondrocranial measures is reduced to $107(24.3 \%)$ at E17.5 suggesting that the similar mean values are coupled with similar patterns of association between chondrocranium and dermatocranium at this age. Of these significant 
differences, 57 (12.9\%) indicate relationships between specific chondrocranium and dermatocranium measures that are mildly to strongly negative in Fgfr $2 \mathrm{C}^{+/+}$mice but mildly to strongly positive in Fgfr2 $c^{C 342 Y /+}$ mice (Fig 3B), while $50(11.3 \%)$ vary from mildly negative to strongly positive in Fgfr2 $\mathrm{c}^{+/+}$ mice but are moderatly to strongly negative in Fgfr2 $\mathrm{C}^{\mathrm{C} 342 \mathrm{Y} / \mathrm{+}}$ mice.

\section{Discussion}

We have provided an improved method for segmentation and visualization of embryonic cranial cartilage by PTA-enhanced microCT imaging and used these data to reveal local and global variations of chondrocranial morphology and its relationship to the dermatocranium in mice carrying an Fgfr2 mutation that is associated with Crouzon syndrome. Our detailed observations of chondrocranial morphology over embryonic time demonstrate the direct effects of the Fgfr2c C342Y mutation on cartilage via chondroblast dysregulation resulting in malformation of the chondrocranium. Fgfr $2 c^{C 342 Y /+}$ mice have a greater amount of cartilage and a chondrocranium that is generally larger and differently shaped relative to their $\mathrm{Fgfr} 2 \mathrm{c}^{+/+}$ littermates at every embryonic age studied (E13.5 to E17.5). The dermatocranial elements of Fgfr2c ${ }^{\mathrm{C} 342 \mathrm{Y} /+}$ mice form on the ectocranial surfaces of cartilage and match the contours and shapes of associated chondrocranial elements contributing to a generally larger and dysmorphic embryonic dermatocranium. Data support our hypothesis that the chondrocranium serves as a scaffold for the later formation of cranial dermal bone (2).

Our findings have significant implications for understanding the role of embryonic cranial cartilage in the initial formation, configuration, and development of cranial dermal bone. The transient nature of the chondrocranium is one reason why so little is known about its role in craniofacial development and mouse models provide an ideal tool for addressing questions pertaining to chondrocranial anatomy and its role in typical development, craniofacial disease, and evolution. Functional explanations for the chondrocranium are appropriate because no modern vertebrate has lost this skeleton during evolution. The ability of cartilage to grow interstitially and by accretion means that the cranial endoskeleton, unlike the cranial dermatoskeleton, can change shape dynamically during embryogenesis acting as a progressively transforming scaffold for developing dermal bone.

Craniosynostosis is a relatively common birth defect, second only to clefts of the lip and palate (13). Most of the work with craniosynostosis-associated genetic variants focus on the bony skull of mouse 
models for craniosynostosis or on human cell lines to demonstrate how specific mutations alter the processes of proliferation, differentiation, apoptosis, and/or polarity of osteoblast lineage cells as they differentiate. Exceptions include a study of $F g f r 2 c^{C 342 Y / C 342 Y}$ mice suggesting that many phenotypic aberrations stem from a primary failure of mesenchymal condensation contributing to aberrant cartilage and bone (43), and observations of enhanced cartilage formation in the trachea of Fgfr2 $^{\mathrm{S} 252 W /+}$ Apert syndrome mice suggesting that abnormal chondrogenesis occurred (51). However, neither study considered the chondrocranium.

Our results indicate that the chondrocrania of $F g f r 2 c^{C 342 Y /+}$ Crouzon syndrome mice are composed of more and larger cartilage cells accompanied by more extracellular matrix, a finding consistent with the significantly larger size and increased thickness. This is the first demonstration that Fgf/Fgfr signaling directly affects chondrocranial shape through changes in chondrocyte activity contributing to the abnormal craniofacial form of Crouzon syndrome. The prenatal bony skulls of Fgfr $2 c^{C 342 Y /+}$ mice are larger than those of their Fgfr2 $c^{+/+}$littermates, while skulls of adult Fgfr $2 c^{C 342 Y /+}$ mice are relatively smaller with domed cranial vaults. These results reveal that a transformative change in skull morphology occurs with disintegration of the transient chondrocranial cartilages, suggesting the significance of the cartilaginous scaffold to later shapes of dermal bones while advancing embryonic cranial cartilage as a potential target for development of early therapies for craniofacial disease.

Our results reveal that the Fgfr2c $\mathrm{C} 342 \mathrm{Y}$ mutation directly targets chondrocytes of the chondrocranium, changing chondrocranial size and shape, and indirectly influencing prenatal dermatocranial size and shape. The Fgfr2c C342Y mutation results in constitutive activation of the receptor associated with up-regulation of osteogenic differentiation $(24,52)$. These regulatory effects on the osteoblast lineage may function at the cellular level prenatally but do not appear to direct the size and shape of dermal bone tissue while the chondrocranium is present. This suggests that the earliest dermal bone in Fgfr2c $\mathrm{C}^{\mathrm{C} 42 \mathrm{Y} /+}$ mice acts non-autonomously, being driven by the mutation's effects on chondrocytes. When chondrocytes of neighboring cartilages disappear however, dermal bone behaves autonomously.

The three main hypotheses we proposed to explain the relationship between chondrocranial cartilage and dermal bone formation include: 1) the Fgfr2c C342Y mutation affects the cranial osteoblast 
lineage but not the chondrocyte series; 2) the mutation affects the chondrocyte series and the osteoblast lineage but the morphological effects of the mutation are dissociated; and 3) the mutation affects the chondrocyte series and the osteoblast lineage and the morphological effects of the mutation emphasize integration of chondrocranial and dermatocranial development. We have shown that changes in chondrocyte size, chondrocyte number, extracellular matrix area, and size and shape of the chondrocranium differentiate the genotypes and clearly point to a direct effect of this mutation on the chondrocyte lineage. Studies of MI reveal an elevated magnitude of association between chondrocranium and dermatocranium at E15.5 for Fgfr2 $\mathrm{C}^{\mathrm{C} 342 \mathrm{Y} /+}$ mice matching previous results that suggested FGF/FGFR signaling as a covariance-generating process in skull development acting to modulates MI intensity (53). The physical and developmental aspect of this integration is mirrored in reduced $\mathrm{MI}$ intensity between chondrocranium and dermatocranium for both genotypes as portions of the chondrocrania begin to dissolve at E17.5.

Our findings are relevant to various fields and challenge traditional thinking about the role of cartilage in the formation of dermal bone. While the association of cartilage is well defined for endochondral ossification, intramembranous ossification is commonly described as mineralization that proceeds without a cartilaginous model. Our data are the first to provide clear evidence of a developmental relationship between cartilaginous elements of the chondrocranium and bones of the dermatocranium. The combination of data presented here and elsewhere $(2,4)$ demonstrates that these relationships underlie normal craniofacial development and dysmorphogenesis, and may offer a mechanistic explanation for the production of cranial variation over evolutionary time. Our study supports the assertion that chondrocranial cartilages function as a scaffold, significantly affecting the position, size, and shape of developing dermal bone. The relationship is temporary however and appears to diminish with the departure of transient cartilages, highlighting the critical, but fleeting impact of chondrocranial cartilage on dermal bone. 


\section{Materials and Methods}

Sample. Mice were produced, sacrificed, and processed in compliance with animal welfare guidelines approved by the Pennsylvania State University Animal Care and Use Committee. Based upon timed mating and evidence of pregnancy, litters were sacrificed and collected as appropriate. PTA staining and alizarin red-alcian blues staining were performed as previously described $(34,54)$.

\section{Imaging Protocols}

MicroCT images for bone and PTA-enhanced microCT images for soft tissue analyses were acquired by the Center for Quantitative Imaging at the Pennsylvania State University (www.cqi.psu.edu) using the General Electric v|tom|x L300 nano/microCT system. This is a dual-tube system with a $300-k V$ microfocus tube for larger specimens and a 180-kV nanofocus tube for smaller specimens. Although specimens may be scanned using either tube, we found the greatest resolution and scan quality were typically produced by the $180-k V$ tube for embryonic specimens and the $300-k V$ tube for postnatal specimens. Image data were reconstructed on a $2024 \times 2024$ pixel grid as a 32-bit volume, but may be reduced to 16 -bit volume for image analysis using Avizo 2020.2 (Thermo Fisher Scientific). Scanning parameters varied from 60$100 \mathrm{kV}$ and $75-170 \mu \mathrm{A}$, to accommodate age group and type of scan performed. Voxel sizes ranged from 6.9 to 15 microns $(\mu \mathrm{m})$ for bone scans and 4.5 to $8 \mu \mathrm{m}$ for PTA-enhanced scans. Three dimensional reconstructions and anatomical landmark data will be available through FaceBase.

\section{Data Collection.}

\section{Segmentation of bone}

A hydroxy apatite (HA) bone phantom was included alongside specimens being scanned for bone. A minimum threshold of $70-100 \mathrm{mg} / \mathrm{cm}^{3}$ partial density HA was used to reconstruct bony isosurfaces in Avizo 2020.2. Specific bone volumes were determined using the Material Statistics module of Avizo. Bone volumes were compared between Fgfr2 $\mathrm{C}^{\mathrm{C} 342 \mathrm{Y} /+}$ mice and $\mathrm{Fgfr}_{2 \mathrm{C}} \mathrm{C}^{+/+}$littermates in IBM SPSS 25 software (IBM) using non-parametric Mann-Whitney $U$ tests due to violations of assumptions of homogeneity or variance and/or normality.

\section{Segmentation of embryonic cartilage}

We previously reported an automatic deep learning based chondrocranium segmentation approach (35). Although deep learning based fully convolutional networks (FCNs) have achieved great successes on 
both generic and biomedical image segmentation (36-38), segmenting chondrocrania in 3D micro-CT images remains a very challenging task. Due to high difficulty in labeling complicated objects (embryonic cranial cartilage) in large 3D micro-CT images to provide sufficient training data for deep learning model training we must resort to sparse annotation (i.e., labeling only a very small subset of $2 \mathrm{D}$ slices in the training set of whole 3D volumes) for training our segmentation model, while still enabling our model to segment the unseen whole volumes (including the delicate and detailed ROIs) with good accuracy. To this end, we developed a new, two-phase approach: (1) automatically segmenting the majority of the chondrocranium with very sparse annotation performed by experts in anatomy that bridges the performance gap compared with full annotation; (2) integrating limited human corrections to fine-tune the model. We present a high-level description of our approach below.

(1) Automatic chondrocranium segmentation with very sparse annotation via uncertainty-guided selftraining. Manual annotation was performed by experts using Avizo 2020.2 (ThermoFisher Scientific). We started with selecting a very sparse subset of $2 \mathrm{D}$ slices (e.g., $2 \%-10 \%$ ) for annotation that represents and covers the unannotated slices of the training volumes well. We then used the annotated slices to train a judiciously designed K-head FCN to predict pseudo-labels (PLs) in the unannotated slices of the training volumes (for bridging the spatial annotation gap) as well as compute the associated uncertainty maps of the PLs (which quantify the pixel-wise prediction confidence or uncertainty). Guided by the uncertainty, we iteratively trained the FCN with PLs and improved its generalization ability in unseen volumes. Moreover, we integrated the segmentation results along three orthogonal planes to further boost the segmentation performance via ensemble learning. Experimental results showed that our approach achieves average Dice scores of $87 \%$ and $83 \%$ in the training and unseen (test) volumes, respectively, with only $3 \%$ annotation of the slices in the training volumes. More details of our approach and validations can be found in (35).

(2) Model fine-tuning via human-aided corrections. The automatic segmentation accuracy in the first phase on extremely difficult ROIs (e.g., Meckel's cartilage and cranial vault) may still not meet the requirement of quantitative analysis, because the model's generalizability is constrained by the highly sparse annotation and the unbalanced amounts of training pixels between easy and difficult regions. Hence, we first evaluated the inadequately segmented regions and manually corrected the algorithm- 
generated predictions, and then combined the annotations thus obtained and PLs to further fine-tune our segmentation model. This process did not incur too much computational costs. Consequently, most specimens were segmented almost perfectly by our model, except for extremely thin, small, or ambiguous regions in certain specimens. Finally, we manually corrected these local errors to generate an accurate chondrocranium model for quantitative analysis.

\section{Landmark Collection}

Three dimensional coordinates of biologically relevant landmarks were collected from slices and isosurfaces created from microCT images of the specimens using Avizo 2020.2. Specimens were digitized twice by the same observer, checked for gross error, and measurement error was minimized by averaging the coordinates of the two trials. A maximum of $5 \%$ error in landmark placement was accepted. Table S1 and Table S2 provide anatomical definitions of all landmarks used.

\section{Statistical Analyses}

Form comparison of embryonic cranial cartilage and bone. To statistically determine shape differences between groups, we used $\operatorname{EDMA}(39,55)$. EDMA converts 3D landmark data into a matrix of all possible linear distances between unique landmark pairs and tests for statistical significance of differences between shapes using a boot-strapped hypothesis testing procedure and non-parametric confidence intervals. We tested for morphological differences between the nasal capsule, lateral walls, and braincase floor of the chondrocranium and the bony skull of Fgfr2 $\mathrm{c}^{\mathrm{C} 342 \mathrm{Y} /+}$ and $\mathrm{Fgfr} \mathrm{C}^{+/+}$mice (39). Use of these subsets in the evaluation of regional shape differences was done to bring the sample size closer to the number of landmarks considered for statistical testing.

Statistical tests for differences of specific linear distances are evaluated by a $90 \%$ confidence interval produced through a non-parametric bootstrapping procedure (55). Rejection of the null hypothesis of similarity for linear distances enables localization of differences to specific dimensions. EDMA analyses were performed using WinEDMA (56) and EDMAinR (57).

Morphological integration. Modern quantitative approaches to the study of integration commonly use matrix correlations and/or covariances to explain how biological structures are organized. Though there are many ways to propose hypotheses of cranial integration based on the analysis of data (see (58) for examples), here, for the first time we study integration within the chondrocranium, within the 
dermatocranium (excluding any landmarks on endochondral skull bones), and between the chondrocranium and dermatocranium. To avoid the use of superimposition to estimate correlation/covariance among traits and differences in these patterns we use linear distances estimated from 3D coordinate locations of biological landmarks (50). By using linear distances, we also circumvent the affine registration (a mapping that includes three translations, three rotations, three scales, and three shears) required to register data from microCT skull images and PTA-enhanced microCT chondrocranial images.

Here we focus on differences in chondrocranium-dermatocranium integration by between-groups statistical comparison of patterns of correlation/covariance in chondrocranium and dermatocranium metrics using a previously published method $(31,50)$. Our analysis provides information about how typical integration of chondrocranium and dermatocranium is altered in the presence of craniosynostosisassociated mutations by comparing integration patterns of $\mathrm{Fgfr}_{2} \mathrm{c}^{\mathrm{C} 342 \mathrm{Y} /+}$ embryos with $\mathrm{Fgfr} 2 \mathrm{C}^{+/+}$littermates. To statistically compare patterns of $\mathrm{Ml}$ of chondrocranium and dermatocranium between groups of mice we used a boot-strap based method $(50,59)$ implemented in MIBoot, a Windows-based software package (56). 3D coordinates of 7 dermatocranial landmarks and 7 chondrocranial landmarks (see Table S2 and Table S1) recorded from microCT and PTA-enhanced microCT, respectively, were used to estimate a total of 861 linear measures (42 unique linear distances among landmarks located on the dermatocranium and 42 unique linear distances estimated between chondrocranial landmarks) that were used in analysis. For each sample, a correlation/covariance matrix was estimated for unique linear distances pairs and a correlation difference matrix was estimated by subtracting the elements of the correlation matrix estimated for the $F g f r 2 c^{C 342 Y /+}$ sample from the corresponding elements of the matrix estimated for the $\mathrm{Fgfr}_{\mathrm{C}} \mathrm{C}^{+/+}$sample used in the age-specific comparison. If the correlation matrices are the same for two samples, then the correlation-difference matrix consists of zeros. If they are not similar, each element of the correlation difference matrix is statistically evaluated using a nonparametric bootstrap approach to estimate confidence intervals $(\alpha=0.10)$. If a confidence interval does not include zero (the expected value under the null hypothesis of similarity), then the null hypothesis of equal associations for that linear distance pair is rejected. Using this method, we statistically compared the correlation patterns 
within the dermatocranium, within the chondrocranium, and between the dermatocranium and chondrocranium between the Fgfr2 $\mathrm{c}^{\mathrm{C} 342 \mathrm{Y} /+}$ Crouzon syndrome mice and $\mathrm{Fgfr} 2 \mathrm{c}^{+/+}$littermates. HIstology. Specimen were fixed overnight in $4 \%$ paraformaldehyde, processed for paraffin-based histology per standard protocol, serially sectioned at $7 \mu \mathrm{m}$ using a manual rotary microtome, stained according to standard safranin-o staining protocol, and imaged using Leica BX50 microscope, DFC450 camera, and LAS-X x-y scanning imaging software (Leica Biosystems, Allendale, NJ). Regions of interest stained with safranin-o were identified and analyzed using Image-J color deconvolution and masks to count stained areas by color (Purple=nuclei, Orange=Cartilage matrix). Non-parametric Mann-Whitney U tests were used to compare genotypes at each age in SPSS 25 software (IBM) as there were violations of assumptions of homogeneity of variance and/or normality.

\section{Acknowledgements}

The authors wish to extend their gratitude to the staff at Pennsylvania State University Center for Quantitative Imaging (https://iee.psu.edu/labs/center-quantitative-imaging) for production of excellent quality images used in this study and Talia Pankratz for assistance in image preparation. This research was supported in part by NICHD/NIH P01HD078233 and NIDCR/NIH R01 DE027677 and NSF CCF1617735.

\section{References}

1. K. V. Kardong, Vertebrates. Anatomy, Function, Evolution, sixth (McGraw-Hill, 2012).

2. K. Kawasaki, J. Richtsmeier, "Association of the chondrocranium and dermatocranium in early skull development" in Building Bones: Early Bone Development Informing Anthropological Inquiry, Cambridge Studies in Biological and Evolutionary Anthropology., C. Percival, J. Richtsmeier, Eds. (Cambridge University Press, 2017), pp. 52-78.

3. K. Kawasaki, J. Richtsmeier, "Appendix to Chapter 3" in Building Bones: Bone Formation and Development in Anthropology, Cambridge Studies in biological and evolutionary anthropology., C. Percival, J. Richtsmeier, Eds. (Cambridge University Press, 2017), pp. 303-315.

4. M. K. Pitirri, K. Kawasaki, J. T. Richtsmeier, It takes two: Building the vertebrate skull from chondrocranium and dermatocranium. Vertebrate Zoology 70, 587-600 (2020).

5. de Beer, G, Development of the vertebrate skull (Oxford University Press, 1937).

6. L. A. Opperman, Cranial sutures as intramembranous bone growth sites. Dev. Dyn 219, 472-485 (2000). 
7. D. T. Farmer, et al., The developing mouse coronal suture at single-cell resolution. Nat Commun 12, 4797 (2021).

8. G. Holmes, et al., Single-cell analysis identifies a key role for Hhip in murine coronal suture development. Nat Comm (in review).

9. S. Iseki, et al., Fgfr2 and osteopontin domains in the developing skull vault are mutually exclusive and can be altered by locally applied FGF2. Development 124, 3375-3384 (1997).

10. K. Flaherty, N. Singh, J. T. Richtsmeier, Understanding craniosynostosis as a growth disorder: Understanding craniosynostosis as a growth disorder. Wiley Interdisciplinary Reviews:

Developmental Biology 5, 429-459 (2016).

11. M. Yu, et al., Cranial Suture Regeneration Mitigates Skull and Neurocognitive Defects in Craniosynostosis. Cell 184, 243-256.e18 (2021).

12. E. Lajeunie, et al., Mutation screening in patients with syndromic craniosynostoses indicates that a limited number of recurrent FGFR2 mutations accounts for severe forms of Pfeiffer syndrome. Eur J Hum Genet 14, 289-298 (2006).

13. Y. Heuzé, G. Holmes, I. Peter, J. T. Richtsmeier, E. W. Jabs, Closing the gap: Genetic and genomic continuum from syndromic to nonsyndromic craniosynostoses. Current genetic medicine reports $\mathbf{2}$, 135-145 (2014).

14. A. Wilkie, Craniosynostosis: genes and mechanisms. Hum. Mol. Genet. 6, 1647-1656 (1997).

15. A. Wilkie, G. Morriss-Kay, J. Radcliffe, O. Ox, Genetics of craniofacial development and malformation. Nature Reviews Genetics 2, 458-468 (2001).

16. C. M. Justice, et al., A genome-wide association study identifies susceptibility loci for nonsyndromic sagittal craniosynostosis near BMP2 and within BBS9. Nat Genet 44, 1360-1364 (2012).

17. Genomics England Research Consortium, et al., SMAD6 variants in craniosynostosis: genotype and phenotype evaluation. Genet Med 22, 1498-1506 (2020).

18. A. Cuellar, et al., Gain-of-function variants and overexpression of RUNX2 in patients with nonsyndromic midline craniosynostosis. Bone 137, 115395 (2020).

19. J. A. C. Goos, I. M. J. Mathijssen, Genetic Causes of Craniosynostosis: An Update. Mol Syndromol 10, 6-23 (2019).

20. T. Maruyama, et al., BMPR1A maintains skeletal stem cell properties in craniofacial development and craniosynostosis. Sci. Transl. Med. 13, eabb4416 (2021).

21. V. P. Eswarakumar, M. C. Horowitz, R. Locklin, G. M. Morriss-Kay, P. Lonai, A gain-of-function mutation of Fgfr2c demonstrates the roles of this receptor variant in osteogenesis. PNAS 101, 12555-12560 (2004). 
22. P. Rutland, et al., Identical mutations in the FGFR2 gene cause both Pfeiffer and Crouzon syndrome phenotypes. Nat Genet 9, 173-176 (1995).

23. M. Oldridge, et al., Mutations in the third immunoglobulin domain of the fibroblast growth factor receptor-2 gene in Crouzon syndrome. Hum Mol Genet 4, 1077-1082 (1995).

24. W. Reardon, et al., Mutations in the fibroblast growth factor receptor 2 gene cause Crouzon syndrome. Nat Genet 8, 98-103 (1994).

25. V. P. Eswarakumar, et al., The Illc alternative of Fgfr2 is a positive regulator of bone formation. Development 129, 3783-3793 (2002).

26. D. Johnson, A. O. M. Wilkie, Craniosynostosis. Eur J Hum Genet 19, 369-376 (2011).

27. H. Zhao, et al., The suture provides a niche for mesenchymal stem cells of craniofacial bones. Nature Cell Biology 17, 386-396 (2015).

28. T. Maruyama, J. Jeong, T.-J. Sheu, W. Hsu, Stem cells of the suture mesenchyme in craniofacial bone development, repair and regeneration. Nat Commun 7, 10526 (2016).

29. S. M. Motch Perrine, et al., Craniofacial divergence by distinct prenatal growth patterns in Fgfr2 mutant mice. BMC Developmental Biology 14, 8 (2014).

30. N. Martínez-Abadías, et al., Tissue-specific responses to aberrant FGF signaling in complex head phenotypes. Dev. Dynam. 242, 80-94 (2013).

31. S. M. Motch Perrine, et al., Integration of brain and skull in prenatal mouse models of Apert and Crouzon syndromes. Frontiers in Human Neuroscience 11, 369 (2017).

32. A. K. Snyder-Warwick, et al., Analysis of a gain-of-function FGFR2 Crouzon mutation provides evidence of loss of function activity in the etiology of cleft palate. Proceedings of the National Academy of Sciences 107, 2515-2520 (2010).

33. C. A. Perlyn, et al., The Craniofacial Phenotype of the Crouzon Mouse: Analysis of a Model for Syndromic Craniosynostosis Using Three-Dimensional MicroCT. The Cleft Palate-Craniofacial Journal 43, 740-747 (2006).

34. K. M. Lesciotto, et al., Phosphotungstic acid-enhanced microCT: Optimized protocols for embryonic and early postnatal mice. Dev. Dynam. 249, 573-585 (2020).

35. H. Zheng, et al., Cartilage Segmentation in High-Resolution 3D Micro-CT Images via UncertaintyGuided Self-training with Very Sparse Annotation" in Proceedings of the Twenty-Third International Conference on Medical Image Computing and Computer Assisted Intervention (MICCAI), A.L. Martel, et al., Eds., 802-812 (Springer International Publishing, Cham, 2020).

36. J. Long, E. Shelhamer, T. Darrell, Fully convolutional networks for semantic segmentation" in Proceedings of the IEEE Conference on Computer Vision and Pattern Recognition (CVPR), 34313440, (IEEE, 2015).37. O. Ronneberger, P. Fischer, T. Brox, U-Net: Convolutional Networks for Biomedical Image Segmentation" in Proceedings of the Eighteenth International Conference on 
Medical Image Computing and Computer Assisted Intervention (MICCAI), N. Navab, et al., 234-241, (Springer International Publishing, Cham, 2015).38. H. Zheng, et al., "HFA-Net: 3D Cardiovascular Image Segmentation with Asymmetrical Pooling and Content-Aware Fusion" in Proceedings of the Twenty-Second Medical Image Computing and Computer Assisted Intervention (MICCAI), D. Shen, et al., Eds., 759-767, (Springer International Publishing, Cham, 2019).39. $\quad$ S. Lele, J. T. Richtsmeier, An invariant approach to statistical analysis of shapes (Chapman \& Hall/CRC, 2001).

40. K. L. Cooper, et al., Multiple phases of chondrocyte enlargement underlie differences in skeletal proportions. Nature 495, 375-378 (2013).

41. N. J. Wilsman, E. S. Bernardini, E. Leiferman, K. Noonan, C. E. Farnum, Age and pattern of the onset of differential growth among growth plates in rats. J. Orthop. Res. 26, 1457-1465 (2008).

42. G. J. Breur, B. A. Vanenkevort, C. E. Farnum, N. J. Wilsman, Linear relationship between the volume of hypertrophic chondrocytes and the rate of longitudinal bone growth in growth plates. J. Orthop. Res. 9, 348-359 (1991).

43. E. Peskett, et al., Analysis of the Fgfr2 ${ }^{\mathrm{C} 342 \mathrm{Y}}$ mouse model shows condensation defects due to misregulation of Sox9 expression in prechondrocytic mesenchyme. Biology Open 6, 223-231 (2017).

44. K. Flaherty, J. Richtsmeier, It's about time: ossification center formation in C57BL/6 mice from E12-E16. JDB 6, 31 (2018).

45. E. Olson, R. Miller, Morphological integration (University of Chicago, 1958).

46. B. Chernoff, P. Magwene, "Afterword. In: Olson E, Miller R, editors. Morphological integration." in Morphological Integration, (University of Chicago, 1999), pp. 319-348.

47. B. Hallgrímsson, et al., Deciphering the Palimpsest: Studying the Relationship Between Morphological Integration and Phenotypic Covariation. Evol Biol 36, 355-376 (2009).

48. K. M. Weiss, The phenogenetic logic of life. Nat Rev Genet 6, 36-45 (2005).

49. S. B. Carroll, Chance and necessity: the evolution of morphological complexity and diversity. Nature 409, 1102-1109 (2001).

50. J. T. Richtsmeier, et al., Phenotypic integration of neurocranium and brain. J Exp Zool Part B 306B, 360-378 (2006).

51. Y. Wang, et al., Abnormalities in cartilage and bone development in the Apert syndrome FGFR2+/S252W mouse. Development 132, 3537-3548 (2005).

52. E. W. Jabs, et al., Jackson-Weiss and Crouzon syndromes are allelic with mutations in fibroblast growth factor receptor 2. Nat Genet 8, 275-279 (1994). 
53. N. Martínez-Abadías, et al., FGF/FGFR Signaling Coordinates Skull Development by Modulating Magnitude of Morphological Integration: Evidence from Apert Syndrome Mouse Models. PLoS ONE 6, e26425 (2011).

54. R. Behringer, M. Gertsenstein, K. Nagy, Manipulating the mouse embryo: a labortory manual (Cold Spring Harbor Laboratory Press, 2014).

55. S. Lele, J. T. Richtsmeier, Euclidean distance matrix analysis: Confidence intervals for form and growth differences. American Journal of Physical Anthropology 98, 73-86 (1995).

56. T. M. Cole III, WinEDMA: Softw. euclidean distance matrix Anal. Version 1.0.1 beta. (Kansas City Univ. Missouri - Kansas City Sch. Med., 2002).

57. P. Solymos, S. Lele, T. Cole III, L. Hu, J. Richtsmeier, EDMAinR: Euclidean Distance Matrix Analysis in $R(2021)$.

58. C. C. Roseman, T. D. Weaver, C. B. Stringer, Do modern humans and Neandertals have different patterns of cranial integration? Journal of Human Evolution 60, 684-693 (2011).

59. T. Cole, S. Lele, Bootstrap-based methods for comparing morphological integration patterns. Am J Phys Anthropol 34, 55 (2002). 